\begin{tabular}{c} 
International Journal of Physical Research, $4(2)(2016) 52-57$ \\
International Journal of Physical Research \\
SPC \\
Website: $\begin{array}{c}\text { www.sciencepubco.com/index.php/IJPR } \\
\text { doi: } 10.14419 / j \text { pr.v4 } 2.6530 \\
\text { Research paper }\end{array}$ \\
\hline
\end{tabular}

\title{
Variational iteration method for studying perihelion precession and deflection of light in General Relativity
}

\author{
V. K. Shchigolev ${ }^{1 *}$ \\ ${ }^{1}$ Department of Theoretical Physics, Ulyanovsk State University, Ulyanovsk, 432000, Russia \\ *Corresponding author E-mail:vkshch@yahoo.com
}

\begin{abstract}
A new approach in studying the planetary orbits and deflection of light in General Relativity (GR) by means of the Variational iteration method (VIM) is proposed in this paper. For this purpose, a brief review of the nonlinear geodesic equations in the spherical symmetry spacetime and the main ideas of VIM are given. The appropriate correct functionals are constructed for the geodesics in the spacetime of Schwarzschild, Reissner-Nordström and Kiselev black holes. In these cases, the Lagrange multiplier is obtained from the stationary conditions for the correct functionals. Then, VIM leads to the simple problem of computation of the integrals in order to obtain the approximate solutions of the geodesic equations. On the basis of these approximate solutions, the perihelion shift and the light deflection have been obtained for the metrics mentioned above.
\end{abstract}

Keywords: Approximate solution; Deflection of light; Perihelion precession; Spherically symmetric spacetime; Variational iteration method.

\section{Introduction}

The perihelion precession of Mercury, the deflection of light by the Sun and the radar echo delay observations are well-known tests for the Schwarzschild solution of GR [1]. It is well known that the geodesics equations in RG are nonlinear, and therefore cannot in general be solved exactly. Only in rare cases, the problem of planetary motion or propagation of the light ray can be solved exactly. For instance, the geodesic equations resulting from the Schwarzschild gravitational metric element are solved exactly by the Weierstraß Jacobi modular form [2].

Mostly, the perihelion precession of planetary orbits and the light deflection based on Einstein's equations had been calculated in different approximations for a general spherically symmetric line element. Most of these approximate methods are based on the existence of a small parameter of the physical origin. At the same time, there are methods for approximate solution of nonlinear differential equations, in which the existence of the small parameter in the equation is not assumed originally. Some examples of these approximate methods are represented by the Homotopy Perturbation Method (HPM) and the Variational Iteration Method, both of which have been proposed by J.-H. He [3]-[6].

More recently, one of these approximate methods, HPM, has been successfully applied to various problems of cosmology and astrophysics [7]-[11]. It could be assumed that the other of the mentioned approximation methods, VIM, can be also successfully applied in GR, particularly for the problems of geodesic motion in the gravitational field of astrophysical objects. In this method, a correction functional is constructed by a general Lagrange multiplier, which can be identified optimally via the variational theory. Being different from the other non-linear analytical methods, such as perturbation methods, this method does not depend on small parameters, such that it can find wide application in non-linear problems without linearization or small perturbations.

It should be noted that the interest to the problems of such a kind is caused not only by the improvement of computational methods, but also due to the theoretical discovery of new, sometimes very exotic, compact objects in the universe. Moreover, due to the great rise of possibilities in observational astronomy for the last few decades, the classical tests of general relativity attract over and again the attention of researchers in various aspects (see, e.g., [12]-[15] and references therein). These studies can concern the gravity effects associated with the modification of the gravitation theory itself, or with some new hypothetical sources of gravity, say, dark energy and dark matter.

A number of astronomical observations strongly confirmed that the Universe is undergoing an accelerated expansion [16]-[20]. In order to explain this phenomenon, an unknown energy component, so called dark energy, has been introduced in the framework of general relativity.The simplest candidate to the role of dark energy is the cosmological constant model which is consistent with most of the current astronomical observations, however it suffers from the cosmological constant problem. It is thus natural to consider other complicated cases. A dynamic scalar field can also serve as the dark energy component such as quintessence, phantom, k-essence, etc. Quintessence is the simplest scalar field dark energy model without having theoretical problems like Laplacian instabilities or ghosts. The energy density and the pressure of quintessence vary with time depending on the scalar field and the potential, which are respectively given by: $\rho_{q}=(1 / 2) \dot{\phi}^{2}+V(\phi)$ and $p_{q}=(1 / 2) \dot{\phi}^{2}-V(\phi)$. One Schwarzschild-like solution related to the quintessence model was found in [21]. This solution describes a spherically symmetric and 
static exterior spacetime surrounded by a quintessence field. The gravitational lensing due to this Schwarzschild-like black hole was investigated in [22]. The consideration of accretion of matter onto Kiselev black hole and some other aspects of problem concerning this kind black hole can be found in [23]-[25].

It is the purpose of the present work to explore He's Variational Iteration Method in studying the orbital motion and the light deflection in the spherically symmetric gravitational field in GR. To this end, we consider the geodesic equations in the spacetime of Schwarzschild, Reissner-Nordström and Kiselev black holes. We demonstrate that VIM leads to the simple problem of computation of the integrals in order to obtain the approximate solutions of the geodesic equations in these cases. Then, by using these approximate solutions, the perihelion shift and the light deflection are obtained for the aforementioned metrics.

\section{Preliminaries}

In this section, we give some basis equations of the geodesics in the spherical symmetry gravitational fields and the method for their solving.

\subsection{Geodesic equations}

Here, we mostly follow Ref. [12] in representing the main equations of geodesic motion in a spherical symmetry spacetime. According to Genaral Relativity [1, 12], in the case of 4-dimensional general spherically symmetric spacetime, its stationary line element can be represented by

$d s^{2}=-f(r) d t^{2}+\frac{d r^{2}}{h(r)}+r^{2}\left(d \theta^{2}+\sin ^{2} \theta d \varphi^{2}\right)$.

Since the perihelion precession and deflection of light are usually treated as the time-like and null geodesics in spacetime, respectively, let us consider the geodesics $\gamma(\tau)$ in the above spherically symmetric spacetime. We set the geodesic $\gamma(\tau)$ expressed in the spherical coordinates $x^{\mu}=(t, r, \theta, \varphi)$ as $x^{\mu}(\tau)$, which satisfy

$\frac{d^{2} x^{\mu}}{d \tau^{2}}+\Gamma_{v \sigma}^{\mu} \frac{d x^{\nu}}{d \tau} \frac{d x^{\sigma}}{d \tau}=0$.

The geodesic $\gamma(\tau)$ can be obtained by solving the above equation. However, taking into account the symmetry of spacetime (1), one could use the following simple way to obtain the geodesic $\gamma(\tau)$. First, we can find that one component of the geodesic $\gamma(\tau)$ can always be chosen as $\theta(\tau)=\pi / 2$, which means that the geodesic can always be chosen to lay in the equatorial plane of the spherically symmetric spacetime. Thus, $t=t(\tau), r=r(\tau), \theta=\pi / 2, \varphi=\varphi(\tau)$. Let us denote the tangent vector of geodesic $\gamma(\tau)$ as $U^{\mu} \equiv d x^{\mu} / d \tau$. For the time-like geodesic, we chose $\tau$ to be the proper time. Hence, from (1) we can obtain

$f(r)\left(\frac{d t}{d \tau}\right)^{2}-h^{-1}(r)\left(\frac{d r}{d \tau}\right)^{2}-r^{2}\left(\frac{d \varphi}{d \tau}\right)^{2}=-k$,

where we have used $\theta=\pi / 2$, and $k=1$ corresponds to the time-like geodesic, while $k=0$ is the null geodesic.

Second, it could be noted that $\xi^{a}=(\partial / \partial t)^{a}$ and $\psi^{a}=(\partial / \partial \varphi)^{a}$ are two Killing vectors in the spherically symmetric spacetime (1). Therefore, there are two conserved quantities along the geodesic $\gamma(\tau)$, the total energy and the angular momentum per unit mass, as follows

$E=-g_{a b} \xi^{a} U^{b}=f(r) \frac{d t}{d \tau}, \quad L=g_{a b} \psi^{a} U^{b}=r^{2} \frac{d \varphi}{d \tau}$.

After inserting (3) into (2), one could obtain

$\left(\frac{d r}{d \tau}\right)^{2}=\frac{h(r)}{f(r)} E^{2}-h(r)\left(k+\frac{L^{2}}{r^{2}}\right)$.
This equation contains only one function $r(\tau)$, and it could be solved in principle. Then, after inserting the solved $r(\tau)$ into (3), the rest components $t(\tau)$ and $\varphi(\tau)$ of geodesic could be finally obtained.

However, it should be noted that the perihelion precession as well as the deflection of light are usually related to the geodesics orbits, i.e. $r(\varphi)$. Therefore, it is convenient to rewrite equation (4) with the help of (3) as

$\left(\frac{d r}{d \varphi}\right)^{2}\left(\frac{L}{r^{2}}\right)^{2}=\frac{h(r)}{f(r)} E^{2}-h(r)\left(k+\frac{L^{2}}{r^{2}}\right)$.

It has been found that the coordinate $u \equiv 1 / r$ is more convenient than $r$ to derive the perihelion precession. Thus, the main equation investigated in our paper could be simply obtained from equation (5) by converting $r$ into $u$

$\left(\frac{d u}{d \varphi}\right)^{2}=\frac{h(u)}{f(u)}\left(\frac{E}{L}\right)^{2}-h(u)\left(\frac{k}{L^{2}}+u^{2}\right)$.

Finally, differentiating the equation (6) with respect to $\varphi$, we get the second-order geodesic equation in the following form

$\frac{d^{2} u}{d \varphi^{2}}=\frac{E^{2}}{2 L^{2}} \frac{d}{d u}\left[\frac{h(u)}{f(u)}\right]-h(u) u-\frac{1}{2}\left(\frac{k}{L^{2}}+u^{2}\right) \frac{d h(u)}{d u}$.

In the simplest case of the metric (1), namely the Schwarzschild metric describing the gravitational field of an uncharged non-rotating star, one gets $f(r)=h(r)=1-2 M / r$, or

$f(u)=h(u)=1-2 M u$.

where $M$ is a mass of the star. Therefore, Eq. (8) for the geodesic can be reduced to

$\frac{d^{2} u}{d \varphi^{2}}+u=k \frac{M}{L^{2}}+3 u^{2} M$

Compared with the case of orbital motion in Newton's gravity

$\frac{d^{2} u}{d \varphi^{2}}+u=\frac{M}{L^{2}}$,

the term $3 u^{2} M$ comes from the correction of general relativity.

In the case of the Reissner-Nordström spacetime of a charged star, we have [12]

$f(u)=h(u)=1-2 M u+Q^{2} u^{2}$,

where $Q$ is the charge. According to (11), the geodesic equation (8) now is replaced by the following one

$\frac{d^{2} u}{d \varphi^{2}}+\left(1+k \frac{Q^{2}}{L^{2}}\right) u=k \frac{M}{L^{2}}+3 u^{2} M-2 Q^{2} u^{3}$.

On the other hand, the geometry of the static spherically symmetric black hole surrounded by a quintessence (or Kiselev spacetime) is given by equation (1) with [21]

$f(u)=h(u)=1-2 M u-\sigma u^{3 w_{q}+1}$,

where $M$ is the mass of the black hole and $\sigma$ is the quintessence equation of state parameter, $w_{q}=p_{q} / \rho_{q}$, that is related to the energy density as follows $\rho_{q}=-3 \sigma w_{q} u^{3\left(1+w_{q}\right)} / 2$.

Substituting (13) into equation (7), one can obtain the following geodesic equations in the gravitational field of Kiselev black hole

$\frac{d^{2} u}{d \varphi^{2}}+u=k \frac{M}{L^{2}}+3 u^{2} M+k \frac{\sigma\left(3 w_{q}+1\right)}{2 L^{2}} u^{3 w_{q}}+\frac{3 \sigma\left(w_{q}+1\right)}{2} u^{3 w_{q}+2}$,

which of course coincides with equation (9) in the absence of quintessence, i.e. when $\sigma=0$. 


\subsection{Description of VIM}

The VIM was developed by He [4]-[6] for solving linear, nonlinear, initial and boundary value problems. To give a general idea of the VIM, we consider the following general non-linear equation:

$L[u(x)]+N[u(x)]=g(x)$,

where $L$ and $N$ are linear and nonlinear operators respectively, and $g(x)$ is a known function. The correct functional for the equation (15) can be written as

$u_{n+1}(x)=u_{n}(x)+\int_{0}^{x} \lambda(s)\left\{L\left[u_{n}(s)\right]+N\left[\tilde{u}_{n}(s)\right]-g(s)\right\} d s$,

where $\lambda$ is a Lagrange multiplier, that can be identified optimally via variational iteration method. Here, $\tilde{u}_{n}$ is considered to be a6 restricted variation which means that $\delta \tilde{u}_{n}=0$. Making the correct functional (16) stationary, yields

$$
\begin{array}{r}
\delta u_{n+1}(x)=\delta u_{n}(x)+\delta \int_{0}^{x} \lambda(s)\left\{L\left[u_{n}(s)\right]+N\left[\tilde{u}_{n}(s)\right]-g(s)\right\} d s \\
=\delta u_{n}(x)+\int_{0}^{x} \delta\left\{\lambda(s) L\left[u_{n}(s)\right]\right\} d s .
\end{array}
$$

Its stationary conditions, $\delta u_{n+1}(x)=0$, can be obtained using integration by parts in Eq. (17) and noticing that $\delta u_{n}(0)=0$. The Lagrange multipliers can be easily and precisely obtained for linear problems. However, for nonlinear problems, it is not as trivial. The nonlinear terms are treated as restricted variations such that the Lagrange multiplier can be determined as a simpler form.

The importance and the very utility of method is endowed with the choice of assumption of considering even highly nonlinear and complicated dependent variables as restricted variables thereby synchronizing the error occurring due to process of finding solution to equation (15) to its minimum magnitude. Eventually, after $\lambda$ is determined as desired, a proper selective function, may it be a linear or otherwise with respect to equation (15) is assumed as an initial approximation for finding next successive iterative function by equation (16) recursively.

The successive approximations $u_{n+1}(x)$ of the solution will be readily obtained upon using the obtained Lagrange multiplier and by using any appropriate function for $u_{0}(x)$. The zeroth approximation $u_{0}(x)$ may be selected by any function that just meets, at least, the initial and boundary conditions. Therefore by starting from $u_{0}(x)$, the exact solution may be obtained as

$u(x)=\lim _{n \rightarrow \infty} u_{n}(x)$

Thus, in applications of VIM to differential equations, one should undertake the following three steps: (i) establishing the correction functional; (ii) identifying the Lagrange multipliers; (iii) determining the initial iteration. For the convergence criteria and error estimates of the VIM, one can refer the reader to [26]-[28].

\section{Studying perihelion precession and deflec- tion of light by VIM}

Now we are going to apply VIM for studying the orbital motion and propagation of the light ray in the metrics mentioned above.

\subsection{Schwarzschild solution}

Comparing equation (15) with the geodesic equation (9) for the Schwarzschild metric, we can write down the correct functional (16) as follows

$u_{n+1}(\varphi)=u_{n}(\varphi)+\int_{0}^{\varphi} \lambda_{\varphi}(\phi)\left[\frac{d^{2} u_{n}}{d \phi^{2}}+u_{n}(\phi)-\frac{k M}{L^{2}}-3 M \tilde{u}_{n}^{2}(\phi)\right] d \phi$,

where $\tilde{u}_{n}(\phi)$ is considered to be a restricted variation. By using the stationary condition for the correct functional (17), we get

$\delta u_{n+1}(\varphi)=\delta u_{n}(\varphi)+\int_{0}^{\varphi} \delta\left\{\lambda_{\varphi}(\phi)\left[\frac{d^{2} u_{n}(\phi)}{d \phi^{2}}+u_{n}(\phi)-\frac{k M}{L^{2}}\right]\right\} d \phi$.

From stationary conditions, $\delta u_{n+1}(\varphi)=0$, and using integration by parts in Eq. (20), on can obtain the following equations for the Lagrange multiplier

$\left\{\begin{array}{l}\lambda_{\varphi}^{\prime}(\phi)_{\mid \phi=\varphi}-1=0, \\ \lambda_{\varphi}(\phi)_{\mid \phi=\varphi}=0, \\ \lambda_{\varphi}^{\prime \prime}(\phi)+\lambda_{\varphi}(\phi)=0 .\end{array}\right.$

These equations can be readily solved that yields the following Lagrange multiplier

$\lambda_{\varphi}(\phi)=\sin (\phi-\varphi)$.

Then, according to (19) and (22), the successive approximations $u_{n}(\phi)$ of the solution can be readily obtained upon using the obtained Lagrange multiplier (22) and by using any appropriate function for $u_{0}(\phi)$.

First, we consider the orbital motion, $\mathrm{k}=1$, starting with the Kepler's orbits

$u_{0}(\varphi)=\frac{M}{L^{2}}(1+e \cos \varphi)$

which is the analytical elliptical solution of (10), already found in Newton's gravity. Here, $e$ is the orbital eccentricity. Inserting $u_{0}(\varphi)$ from (23) and $\lambda$ from (22) in equation (19), we obtain the following first order approximation

$u_{1}(\varphi)=\frac{M}{L^{2}}(1+e \cos \varphi)-\frac{3 M^{3}}{L^{4}} \int_{0}^{\varphi} \sin (\phi-\varphi)(1+e \cos \varphi)^{2} d \phi$,

which gives us the approximate solution $u \approx u_{1}$ of equation (9) as

$$
\begin{aligned}
u(\varphi)=\frac{M}{L^{2}}(1+e \cos \varphi)+\frac{M^{3}}{L^{4}}[ & 3+2 e^{2}+3 e \varphi \sin \varphi \\
& \left.-e^{2} \cos ^{2} \varphi-\left(3+e^{2}\right) \cos \varphi\right]
\end{aligned}
$$

It is interesting that this solution exactly coincides with the similar one obtained in Ref. [8] by the Homotopy Perturbation Method. Comparing our result (24) with the corresponding approximate formula in Ref. [12], one can conclude that this solution is different in the term $-\left(M^{3} / L^{4}\right)\left(3+e^{2}\right) \cos \varphi$. The magnitude of the shift angle can be obtained from the condition $u^{\prime}(\varphi)=0$ in the perihelion, and $\varphi=2 \pi+\Delta \varphi$, assuming that $\Delta \varphi \ll 1$ [8]. As applied to the approximate solution (26), this method gives the following value of the perihelion shift

$\Delta \varphi=6 e \pi \frac{M^{2}}{L^{2}}\left[e-3(1+e)^{2} \frac{M^{2}}{L^{2}}\right]^{-1}$,

which is differ from the classical $\Delta \varphi=6 \pi M^{2} / L^{2}$ but results in the same expression for $M^{2} / L^{2} \ll 1$. 
Let us consider the light propagation near the Schwarzschild black hole according to Eq. (16) with $k=0$. Now, the null approximation is a straight line expressed in polar coordinates

$u_{0}(\varphi)=\frac{1}{l} \sin \varphi$

where $l$ is a constant. Substitution of (26) and $\lambda_{\varphi}(\phi)$ (see equation (22)) along with $k=0$ into (19) yields the first approximation as follows

$u_{1}(\varphi)=\frac{1}{l} \sin \varphi-\frac{3 M}{l^{2}} \int_{0}^{\varphi} \sin (\phi-\varphi) \sin ^{2} \phi d \phi$

This immediately leads to the approximate solution $u \approx u_{1}$ for the light path given by

$u(\varphi)=\frac{1}{l} \sin \varphi+\frac{M}{l^{2}}(1-\cos \varphi)^{2}$,

which is exactly the same as obtained in [12]. Therefore, the deflection angle of light $\beta$, which can be obtained from the equation $u(\pi+\beta)=0$ and the following approximations

$\sin (\pi+\beta) \approx-\beta, \quad \cos (\pi+\beta) \approx-1$.

is as follows

$\beta=\frac{4 M}{l}$

that is just the well-known deflection angle of light in Schwarzschild spacetime.

Let us obtain a more accurate approximation for the solution of equation (9). For this purpose, we substitute $u_{1}$ from (27) into equation (19) along with $n=1$ and $k=0$. As a result, we have

$$
\begin{aligned}
& u_{2}(\varphi)=\frac{1}{l} \sin \varphi+\frac{M}{l^{2}}(1-\cos \varphi)^{2} \\
& -\frac{3 M^{2}}{l^{3}} \int_{0}^{\varphi} \sin (\phi-\varphi)\left[2 \sin \phi(1-\cos \phi)^{2}+\frac{M}{l}(1-\cos \phi)^{4}\right] d \phi
\end{aligned}
$$

After integration, we arrive at the following approximation $u \approx u_{2}$ :

$$
\begin{aligned}
& u(\varphi)=\frac{1}{l} \sin \varphi+\frac{M}{l^{2}}(1-\cos \varphi)^{2} \\
&+\frac{M^{2}}{4 l^{3}}\{2 \sin \varphi-[(3 \cos \varphi-16) \sin \varphi+15 \varphi] \cos \varphi\} \\
&+\frac{M^{3}}{10 l^{4}}\left[166-2 \cos ^{4} \varphi+15 \cos ^{3} \varphi-68 \cos ^{2} \varphi\right. \\
&-111 \cos \varphi-105 \varphi \sin \varphi] .
\end{aligned}
$$

Neglecting for simplicity the last term in equation (30), that is proportional to $M^{3} / l^{4}$, one can obtain the deflection angle as

$\beta=\frac{4 M}{l} \times \frac{\left(1+\frac{15 \pi}{8} \frac{M}{l}\right)}{\left(1-8 \frac{M^{2}}{l^{2}}\right)} \approx \frac{4 M}{l}\left(1+\frac{15 \pi}{8} \frac{M}{l}+8 \frac{M^{2}}{l^{2}}\right)$,

which gives the extra terms of the higher order to the deflection angle compared to (29).

\subsection{Reissner-Nordström spacetime}

In this case, we prefer to write down the correct functional (16) for equation (12) as follows

$$
\begin{aligned}
u_{n+1}(\varphi)=u_{n}(\varphi) & +\int_{0}^{\varphi} \lambda_{\varphi}(\phi)\left[\frac{d^{2} u_{n}}{d \phi^{2}}+u_{n}(\phi)-\frac{k M}{L^{2}}\right. \\
& \left.+k \frac{Q^{2}}{L^{2}} \tilde{u}_{n}(\phi)-3 M \tilde{u}_{n}^{2}(\phi)+2 Q^{2} \tilde{u}_{n}^{3}(\phi)\right] d \phi .
\end{aligned}
$$

Comparing this equation with (19), we can conclude that the stationary condition for the correct functional (17) has the same form (20) as it is in previous subsection. Therefore, once again we obtain the same Lagrange multiplier, $\lambda_{\varphi}(\phi)=\sin (\phi-\varphi)$. Using this multiplier and the null approximation (23), one can obtain the first approximation for the orbits (that is, $\mathrm{k}=1$ ) from (32) as follows

$$
\begin{gathered}
u_{1}(\varphi)=\frac{M}{L^{2}}(1+e \cos \varphi)+\int_{0}^{\varphi} \sin (\phi-\varphi)\left[\frac{M Q^{2}}{L^{4}}(1+e \cos \phi)\right. \\
\left.-\frac{3 M^{3}}{L^{4}}(1+e \cos \phi)^{2}+\frac{2 Q^{2} M^{3}}{L^{6}}(1+e \cos \phi)^{3}\right] d \phi,
\end{gathered}
$$

which can be integrated, using the Maple package for example, resulting in the approximate solution, $u \approx u_{1}$, for equation (12) given by

$$
\begin{aligned}
u(\varphi) & =\frac{M}{L^{2}}(1+e \cos \varphi)+\frac{M^{3}}{L^{4}}\left[3+2 e^{2}-2\left(1+2 e^{2}\right) \frac{Q^{2}}{L^{2}}\right. \\
& -\frac{Q^{2}}{M^{2}}+\left(1-\frac{Q^{2}}{L^{2}}+\frac{Q^{2}}{6 M^{2}}-e^{2} \frac{Q^{2}}{4 L^{2}}\right) 3 e \varphi \sin \varphi \\
& -\left(3+e^{2}+\left(e^{3}-8 e^{2}-8\right) \frac{Q^{2}}{4 L^{2}}-\frac{Q^{2}}{M^{2}}\right) \cos \varphi \\
& \left.-\left(1-2 \frac{Q^{2}}{L^{2}}\right) e^{2} \cos ^{2} \varphi+\frac{Q^{2}}{4 L^{2}} e^{3} \cos ^{3} \varphi\right]
\end{aligned}
$$

The shift angle can be obtained from the same conditions $u^{\prime}(\varphi)=0$ and and $\varphi=2 \pi+\Delta \varphi$, applied to the approximate solution (34). Thus, we get the following value of the perihelion shift

$\Delta \varphi \approx 6 e \pi \frac{M^{2}}{L^{2}}\left(1+\frac{Q^{2}}{6 M^{2}}-\left(4+e^{2}\right) \frac{Q^{2}}{4 L^{2}}\right)\left[e-3(1+e)^{2} \frac{M^{2}}{L^{2}}\right]^{-1}$,

Then, we consider the light deflection near Reissner-Nordström black hole according to Eq. (32) with $k=0$. Again, the null approximation is a straight line expressed in polar coordinates by (26).

Substituting (26) along with (22) and $k=0$ into (32), one can get the first approximation as

$$
\begin{aligned}
u_{1}(\varphi)=\frac{1}{l} \sin \varphi-\frac{3 M}{l^{2}} \int_{0}^{\varphi} \sin (\phi & -\varphi) \sin ^{2} \phi d \phi \\
& +\frac{2 Q^{2}}{l^{3}} \int_{0}^{\varphi} \sin (\phi-\varphi) \sin ^{3} \phi d \phi,
\end{aligned}
$$

from which we have the approximate solution $u \approx u_{1}$ for the light path given by

$$
\begin{aligned}
u(\varphi)=\frac{1}{l} \sin \varphi+\frac{M}{l^{2}}( & 1-\cos \varphi)^{2} \\
& -\frac{Q^{2}}{4 l^{3}}\left[\left(\cos ^{2} \varphi+2\right) \sin \varphi-3 \varphi \cos \varphi\right] .
\end{aligned}
$$

From the latter, the deflection angle of light $\beta$ can be obtained with the help of approximations (28) as follows

$\beta=\frac{4 M}{l}-\frac{3 \pi Q^{2}}{4 l^{2}}$. 
It should be noted that the deflection angle (38) was obtained only by the simple approximation (36), while a similar expression in Ref. [12] was obtained by a further simplification of the corresponding formula.

\subsection{Spacetime of Kiselev black hole}

In this case, we can write down the correct functional (16) for Eq. (12) as follows

$$
\begin{aligned}
& u_{n+1}(\varphi)=u_{n}(\varphi)+\int_{0}^{\varphi} \lambda_{\varphi}(\phi)\left[\frac{d^{2} u_{n}}{d \phi^{2}}+u_{n}(\phi)-\frac{k M}{L^{2}}\right. \\
& \left.-3 M \tilde{u}_{n}^{2}(\phi)-k \frac{\sigma\left(3 w_{q}+1\right)}{2 L^{2}} \tilde{u}^{3 w_{q}}-\frac{3 \sigma\left(w_{q}+1\right)}{2} \tilde{u}^{3 w_{q}+2}\right] d \phi
\end{aligned}
$$

Unfortunately, this method has a difficult computational problem in the case of arbitrary quintessence EOs parameter $w_{q}$. Therefore, we are going to proceed with more detail considering Kiselev black hole with quintessential parameters $w_{q}=-1 / 3$ when the resulting formulae can be given in a relatively simple form. Substituting this EoS along with $k=1$ into equation (39) and comparing the latter with (19), we again obtain the Lagrange multiplier $\lambda_{\varphi}(\phi)=\sin (\phi-\varphi)$. By means of this multiplier and the null approximation (23), one can obtain the first approximation for the orbits followed from (39) as

$$
\begin{array}{r}
u_{1}(\varphi)=\frac{M}{L^{2}}(1+e \cos \varphi)-\frac{3 M^{3}}{L^{4}} \int_{0}^{\varphi} \sin (\phi-\varphi)\left[(1+e \cos \phi)^{2}\right. \\
\left.+\frac{\sigma}{3} \frac{L^{2}}{M^{2}}(1+e \cos \phi)\right] d \phi
\end{array}
$$

which can be easily integrated resulting in the approximate solution, $u \approx u_{1}$, for equation (14) given by

$$
\begin{aligned}
u(\varphi)= & \frac{M}{L^{2}}(1+e \cos \varphi)+\frac{M^{3}}{L^{4}}\left[3+2 e^{2}+\sigma \frac{L^{2}}{M^{2}}-e^{2} \cos ^{2} \varphi\right. \\
& \left.+3 e\left(1+\frac{\sigma}{6} \frac{L^{2}}{M^{2}}\right) \varphi \sin \varphi-\left(3+e^{2}+\sigma \frac{L^{2}}{M^{2}}\right) \cos \varphi\right]
\end{aligned}
$$

The approximate magnitude of the shift angle is followed from the condition $u^{\prime}(\varphi)=0$ in the perihelion, and $\varphi=2 \pi+\Delta \varphi$. As applied to the approximate solution (40), this method gives the following value of the perihelion shift

$\Delta \varphi=6 e \pi \frac{M^{2}}{L^{2}}\left(1+\frac{\sigma}{6} \frac{L^{2}}{M^{2}}\right)\left[e-(1+e) \sigma-3(1+e)^{2} \frac{M^{2}}{L^{2}}\right]^{-1}$

which coincides with (25) at $\sigma=0$. Provided that $\sigma \ll 1$ and $\left(M^{2} / L^{2}\right) \ll 1$, this equation is substantially simplified up to

$\Delta \varphi=6 \pi \frac{M^{2}}{L^{2}}+\pi \sigma$

Hence, the presence of quintessence gives the extra angle $\pi \sigma$ to the perihelion shift.

Finally, let us consider the light deflection by Kiselev black hole according to Eq. (39) with $k=0$ and $w_{q}=-1 / 3$. We again take the straight line (26) as the initial approximation.

Substituting (26) and (22) into (39), we can obtain the first approximation,

$u_{1}(\varphi)=\frac{1}{l} \sin \varphi-\frac{1}{l} \int_{0}^{\varphi} \sin (\phi-\varphi)\left(\frac{3 M}{l} \sin ^{2} \phi+\sigma \sin \phi\right) d \phi$

from which we have the approximate solution $u \approx u_{1}$ given by

$u(\varphi)=\frac{1}{l} \sin \varphi+\frac{M}{l^{2}}(1-\cos \varphi)^{2}+\frac{\sigma}{2 l}(\sin \varphi-\varphi \cos \varphi)$.
From this equation, the deflection angle of light $\beta$ can be obtained with the help of approximations (28) as follows

$\beta=\frac{4 M}{l}+\frac{\pi \sigma}{2}$

From this equation, one could conclude that an additional angle to the deflection due to quintessence can be up to $\pi / 2$ at $\sigma \rightarrow 1$. Of course, it is not true since the approximate equation (28) for the deflection angle $\beta$ is not valid in this case. Nevertheless, equation (43) yields the general trend in the dependence of the deflection angle regarding $\sigma$.

\section{Conclusion}

Thus, in this work we have considered a simple analytical computation of the perihelion precession and the deflection of light in General Relativity with the help of Variational Iteration Method developed by Dr. He [3]. First of all, we have studied the examples of geodesic motion in the Schwarzschild and Reissner-Nordström metrics, in order to approbate VIM in the problems of planetary motion and deflection of light, and present the main steps in solving by this method. Then, we have applied VIM for solving the geodesic equations in the spacetime of Kiselev black hole. As a result, we have obtained the perihelion shift (41) and the light deflection angle (43) due to the gravity of Kiselev black hole subject to the EoS $w_{q}=-1 / 3$.

It is interesting that the results obtained by using VIM in most cases coincide with the corresponding results obtained by the perturbation theory or HPM. However, here we have avoided discussion of the smallness of the physical parameters, the need for which may arise only in solving the problem of convergence of the approximate solutions. An important advantage of this method is the simplicity of obtaining approximate solutions to the problems considered, and also the possibility of simple obtaining the next approximation by repeated applications of the iterative equations. We would like to express the hope that VIM is able to find even more applications in the field of astrophysics and cosmology than have been used here.

\section{References}

[1] S. Weinberg. Gravitation and Cosmology: Principles and Applications of The General Theory of Relativity, John Wiley. Press, New York 1972.

[2] G. V. Kraniotis, S. B. Whitehouse, "Compact calculation of the Perihelion Precession of Mercury in General Relativity, the Cosmolog ical Constant and Jacobi's Inversion problem", Classical and Quan tum Gravity, 20 (2003), 4817-4835. http://dx.doi.org/10.1088/0264 9381/20/22/007

[3] J.-H. He, "Homotopy perturbation technique", Computer Methods in Applied Mechanics and Engineering, 178 (1999), 257-262. http://dx.doi.org/10.1016/S0045-7825(99)00018-3

[4] J. H. He, "Variational iteration method - a kind of non-linear analytical technique: some examples", International Journal of Non-Linear Mechanics, 34(4) (1999), 699-708. http://dx.doi.org/10.1016/S0020 7462(98)00048-1

[5] J. H. He, "Variational iteration method for autonomous ordinary differential systems", Applied Mathematics and Computation, 114(2-3) (2000), 115-123. http://dx.doi.org/10.1016/S0096-3003(99)00104-6

[6] J. H. He, "Variational iteration method-Some recent results and new interpretations", Journal of Computational and Applied Mathematics, 207(1) (2007), 3-17. http://dx.doi.org/10.1016/j.cam.2006.07.009

[7] V. Shchigolev, "Homotopy Perturbation Method for Solving a Spatially Flat FRW Cosmological Model", Universal Journal of Applied Mathematics, 2(2) (2014), 99-103. http://dx.doi.org/10.13189/ujam.2014.020204

[8] V. Shchigolev, "Analytical Computation of the Perihelion Precession in General Relativity via the Homotopy Perturbation Method", Universal Journal of Computational Mathematics, 3(4) (2015), 45-49. http://dx.doi.org/10.13189/ujcmj.2015.030401

[9] V. K. Shchigolev, "Calculating Luminosity Distance versus Redshift in FLRW Cosmology via Homotopy Perturbation Method", http://arxiv.org/abs/1511.07459

[10] F. Rahaman, S. Ray, A. Aziz, S. R. Chowdhury, D. Deb, Exact Radiation Model For Perfect Fluid Under Maximum Entropy Principle, http://arxiv.org/abs/1504.05838 
[11] Abdul Aziz, Saibal Ray, Farook Rahaman, "A generalized model for compact stars", European Physical Journal C, 76 (2016), 248 http://dx.doi.org/10.1140/epjc/s10052-016-4090-0

[12] Ya-Peng Hu, Hongsheng Zhang, Jun-Peng Hou, and Liang-Zun Tang. "Perihelion Precession and Deflection of Light in the General Spherically Symmetric Spacetime", Advances in High Energy Physics, Volume 2014, Article ID 604321, 7 pages. http://dx.doi.org/10.1155/2014/604321

[13] Hideyoshi Arakida, "Note on the Perihelion/Periastron Advance Due to Cosmological Constant", International Journal of Theoretical Physics, 52 (2013), 1408-1414. http://dx.doi.org/10.1007/s10773-012-1458-2

[14] Christian Magnan, "Complete calculations of the perihelion precession of Mercury and the deflection of light by the Sun in General Relativity", http://arxiv.org/abs/0712.3709

[15] A.S. Fokas, C.G. Vayenas, D. Grigoriou, "Analytical computation of the Mercury perihelion precession via the relativistic gravitational law and comparison with general relativity", http://arxiv.org/abs/1509.03326

[16] A. G. Riess, et al. , "Observational Evidence from Supernovae for an Accelerating Universe and a Cosmological Constant", Astronomical Journal, Vol. 116 (1998), 1009. http://dx.doi.org/10.1086/300499

[17] S. Perlmutter, et al., "Measurements of Omega and Lambda from 42 High-Redshift Supernovae", Astrophysical Journal, Vol. 517 (1999), 565. http://dx.doi.org/10.1086/307221

[18] D. N. Spergel, et al., "First-Year Wilkinson Microwave Anisotropy Probe (WMAP) Observations: Determination of Cosmological Parameters", Astrophysical Jounal Supplement Series, 148 (2003), 175- 194 http://dx.doi.org/10.1086/377226

[19] M. Tegmark, M. A. Strauss, et al., "Cosmological parameters from SDSS and WMAP”, Physical Review D, 69 (2004), 103501. http://dx.doi.org/10.1103/PhysRevD.69.103501

[20] S. W. Allen, R. W. Schmidt, H. Ebeling, et al. , "Constraints on dark energy from Chandra observations of the largest relaxed galaxy clusters", Monthly Notices of the Royal Astronomical Society, 353 (2004), 457-467. http://dx.doi.org/10.1111/j.1365-2966.2004.08080.x

[21] V. V. Kiselev, "Quintessence and black holes", Classical and Quantum Gravity, 20 (2003), 1187-1198. http://dx.doi.org/10.1088/02649381/20/6/310

[22] Azka Younas, Mubasher Jamil, Sebastian Bahamonde, Saqib Hussain, "Strong Gravitational Lensing by Kiselev Black Hole", Physical Review D, 92 (2015), 084042. http://dx.doi.org/10.1103/PhysRevD.92.084042

[23] Lei Jiao and Rong-Jia Yang, "Accretion onto a Kiselev black hole", http://arxiv.org/abs/1605.02320

[24] Bushra Majeed, Mubasher Jamil, Parthapratim Pradhan, "Thermodynamic Relations for Kiselev and Dilaton Black Hole", Advances in High Energy Physics, 2015 (2015), 124910. http://dx.doi.org/10.1155/2015/124910

[25] Ibrar Hussain, Sajid Ali, "Marginally Stable Circular Orbits in Schwarzschild Black Hole Surrounded by Quintessence Matter", http://arxiv.org/abs/1601.01295

[26] M. Tari and M. Dehghan, "On the Convergence of He's Variational Iteration Method", Journal of Computational and Applied Mathematics, 207(1) (2007), 121-128. http://dx.doi.org/10.1016/j.cam.2006.07.017

[27] J. I. Ramos, "On the Variational Iteration Method and Other Iterative Techniques for Nonlinear Differential Equations," $A p$ plied Mathematics and Computation, 199(1) (2008), 39-69. http://dx.doi.org/10.1016/j.amc.2007.09.024

[28] Ernest Scheiber, "On the Convergence of the Variational Iteration Method", http://arxiv.org/abs/1509.01779 